the rectum every four hours. In the afternoon Dr. Gee again kindly saw the case with me and confirmed the diagnosis of meningitis, holding out but little hope of ultimate recovery. Owing to the extreme cardiac debility he would not, however, recommend any other line of treatment. Towards the evening the condition of the patient became more alarming, the bromide of potassium having little or no effect. She complained somewhat of pressure over the parietal bones. There was ceaseless noisy delirium, the pulse being over 180 and running, the pupils widely dilated and equal, and respiration 30 .

21 st. - At 5 A.M. I decided to give a hypodermic injection of $\frac{1}{80}$ gr. of hyoseyamine. In half an hour she was almost comatose, with an extremely small pulse, respiration being unaffected. Epileptoid convulsions came on, lasting an hour, and at 7 A.M. she passed into a semi-conscious state and appeared at the point of death. She again rallied after a hypodermic injection of brandy and strychnia, and subsequently she was able to take food by the mouth for the first time for some hours. Her pulse was now 110 and the temperature was subnormal. During the day she was quite conscious and sensible, but in a very nervous condition. Towards evening the former symptoms of maniacal excitement again came on, with a fresh rise of temperature, which was $101^{\circ}$. Dr. Ferrier kindly saw her with me at 9.30 P.M., agreeing in the diagnosis of meningitis, but he thought that optic neuritis was not present. Counter-irritation was carried out behind the ears and on the scalp with croton oil liniment, and half a drachm of paraldehyde with ten grains of antipyrin were injected into the rectum. After an hour she became semi-comatose and cyanosed, with very rapid breathing and the pulse incountable. A hypodermic injection of fifteen minims of sulphuric ether caused a fresh rally.

22nd.-About 9 A. Mr. another epileptoid seizure took place, with external deviation of the left eye. At midday she partly regained consciousness and died at 12.20 P.M.

From the 18th I was unable to obtain a specimen of the arine, everything being passed under her.

South Kensington.

\section{COMPOUND COMMINUTED FRACTURE OF THE SKULL, WITH INJURY TO BRAIN ; RECOVERY.}

By W. C. D. PRENDERGast, M.D., M.Ch. R.U.I.

ON January 4th, 1889, I was summoned to see a young man aged eighteen years, a taker-off at the Ubberley Pit, near Hanley. Whilst working at the bottom of a dip he was struck, through an empty waggon hook slipping off, by a Loaded waggon weighing $18 \mathrm{cwt}$. The length of the coal dip was ten yards, with a fall of eighteen inches to the yard. He was conveyed home, where I first saw him at 7 A.M. He was completely unconscious and bleeding profusely. On examining him I found that the scalp from ear to ear had been lacerated and stripped back, a portion of the periosteum adhering. A part of the skull-cap, comprising a fragment of the frontal bone, together with that portion of the middle and upper third of both parietal bones, was fractured and detached from the surface of the brain, remaining attached behind by a strip of bone at the parietal eminence. A small portion of brain matter, with adherent pia mater, was also discovered in the wound. Both body and wound were grimy with finely pulverised coal dust. The wound was washed carefully with a carbolised solution. The bones were replaced over the brain, some small fragments of bone being removed; the scalp, with the torn periosteum, was brought together with about twenty sutures. On the left side, at the anterior superior angle of the parietal bone, a portion of the scalp was completely torn away, leaving no possible chance of reflecting the scalp over the fractured bone. Compresses of carbolised 3olution and a head bandage completed the dressings. There was continuous oozing from the torn vessels of the diploë, so much so that, owing to the difficulty of controlling this form of bleeding, death from hæmorrhage seemed likely to ensue. Some trouble was experienced in cleansing the wound, on account of the presence of the fine coal dust. I have invariably found, however, that its presence does not interfere materially with the union of wounds. Nearly all incised wounds of colliers inflicted whilst at work heal by first intention. A tattoo mark is left which distinguishes the collier from all other workmen.

On Jan. 5th there was a slight return to consciousness, as was evidenced by the ability to swallow a little soda-and-milk, which was ordered, together with small doses of brandy, to rally the patient from the shock and the persistent hæmorrhage. There was no vomiting. The pupils were slightly dilated, but were acting.

Jan. 6th.-The dressings were removed and the hæmorrhage had nearly ceased. There was slight delirium at night, vomiting being absent. The wound was dressed with carbolised oil and absorbent cotton wool.

7th.--Delirium at night was slight. Food was taken better and the bowels were evacuated. Temperature $99.2^{\circ}$; pulse 126.

8th. - Temperature $99 \cdot 4^{\circ}$; pulse 112 . He slept better and food was taken well.

9th. - A portion of the scalp wound had healed by first intention. Five sutures were removed.

11th. - Suppuration showed itself at one or two points.

12th. - The remaining sutures were removed, the appetite was better, and sleep was prolonged without delirium.

13th. - The major portion of the scalp wound had healed by first intention. In one or two places, however, the bone was exposed; these in the course of a few days were covered with soft velvet-like granulations. From the points mentioned under Jan. 11th there was a continuous oozing of pus, pointing to the presence of some decaying fragments of bone. The case after this was treated as one of necrosis with suppuration.

About the end of January pain was complained of. There were a rise in the temperature and some slight vomiting. The scalp was shaved on Feb. 14th and at one spot, where the pain was greatest, an incision was made down to the bone. Some matter issued, and on probing a loose but not detached piece of bone was felt. The incision was kept open.

March 26th. - I cut down on the detached bone and removed it. It was a fragment of the frontal bone.

April 2nd.-I removed the remaining loose fragments of the frontal bone.

25 th. - I cut down and removed a portion of the parietal bone, including both the internal and external table, measuring two inches and a half in length and one inch and a quarter in breadth.

May 8th. - Another fragment of the parietal bone was removed, corresponding to the left anterior superior angle, and comprising both the internal and external surfaces.

June 10th. - A large fragment of the parietal bone was removed, measuring two inches in length and three-quarters of an inch in breadth, the major portion being the external surface only.

July 2nd. - Some smaller fragments were removed.

27th. - A large fragment of the parietal bone was removed. This was the last operation. The whole wound, together with the incisions, healed continuously and no further suppuration was apparent. The patient began to go out, rapidly regained strength, and about the end of August was able to resume work. Since that time he has enjoyed excellent health. A few months ago a few spicula of bone were thrown out. At present the whole tract is healed and the patient regularly performs his arduous work as a miner. On stooping he complains of a slight sense of giddiness.

Remarks. - The above case illustrates remarkably the possibility of recovery after the most extensive injury to the head. In this case there was an extensive scalp wound, destruction of a portion of the scalp and extensive fracture, with exfoliation of a large surface of bone. One vital factor, however, was absent; there was no pressure on the brain from depression of the cranial bones. Another point worth noticing-although this may not be a typical case to illustrate it by-is that fine coal dust, instead of being a deterrent to the healing of wounds, appears to have the opposite effect. Hanley.

The Matithews Duncan Migmorial.-A meeting of the subscribers to this memorial will be held on Monday next, when the report of the committee will be presented. It appears that the sum of $£ 6319 s$. has already been received and has been invested, after certain necessary expenses bad been paid, in the names of three trustees. The first award of the gold medal and prizes will be made in June next. 


\section{A CASE OF SUPPURATION IN THE ETHMOIDAL CELLS.}

BY W. R. H. STEWART, F.R.C.S. \& L.R.C.P. EDIN., M.R.C.S. \&c

AURAL SURGEON TO THE GREAT NORTHERN CENTRAL HOSPITAL AND SURGEON TO THE LONDON THROAT HOSPITAL.

A MARRIED woman was sent to me at the Great Northern Central Hospital by my colleague, Mr. Morton, in the beginning of 1891 . She told the following story. Twenty years ago she contracted scarlet fever. When convalescing a large abscess formed in the corner of the right eye, and she also experienced slight deafness. The abscess in the eye burst without surgical aid, and both ears discharged incessantly for a week and have done so from time to time ever since. She experiensed no further trouble from the eye for ten years, but suffered occasionally from very severe

FIG. 1.

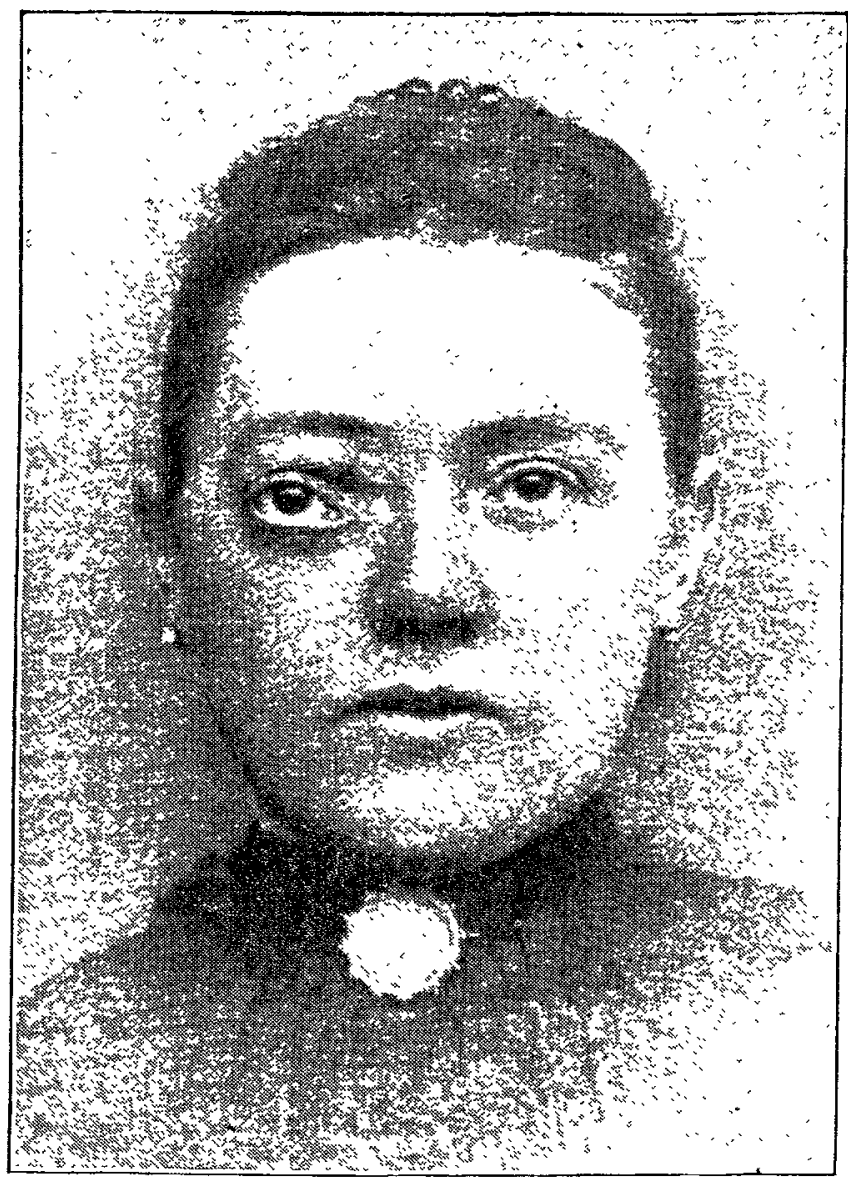

headaches. When out one day she suddenly felt a most violent pain, which lasted for a week, and during that time she could not sleep or lie down and was at times unconscious. She consulted an oculist, who told her that she had a tumour at the back of the eye. He incised a hard substance in the corner of the eye, when a large quantity of pus came away. Eighteen months afterwards the eye was again very painful and the swelling was once more opened, but with little relief. The pain in the head was very severe for some time, and the swelling was opened a third time. Since then, nine years ago, the pain in the head from time to time had been almost unbearable, lasting from a few hours to two or three days. The swelling of the forehead and temple was always much inflamed whilst the pain lasted. Five years ago, after a very bad attack of pain, the patient used a strong lotion, as hot as possible; this brought away quantities of pus down the nose into the throat. Large masses had since come down into the throat. During the last few months the substance in the corner of the eye had become larger. The eye itself was more prominent, the attacks of pain were more frequent, and affected the teeth so much at times that she could not bite anything. The parts seemed numb when not painful.
When I saw the patient two years ago the right eye was pushed outwards and downwards, protruding to a considerable extent, and there was a round swelling in the interior and superioy corner of the orbit (Fig. 1). The canaliculi had been slit up and there was some discharge oozing from them. $O B$ examining the nose a large swelling occupied the place of the right middle turbinate bone which to the probe felt hard and tense. The naso-pharynx was free. Under an anæsthetic 1 punctured the turbinate enlargement with a trocar and on inserting my little finger into the nostril the tumour crackled up before it and I was enabled to pass the finger on into the orbit. I therefore made a free incision into the internal and superior corner of the orbit and found a large amount of dean bone and the orbit full of stringy pus which was pushing out the eyeball; the pus had also hollowed out a cavity in the direction of the frontal sinus into which I could insert the tip of my little finger. All dead bone was removed and the orbit washed out for about a quarter of an hour with a warm boric acid solution until the pus and débris had been entirely cleared out. A large drainage-tube was then inserted into the nostril and brought out through the wound at the corney

Fig. 2

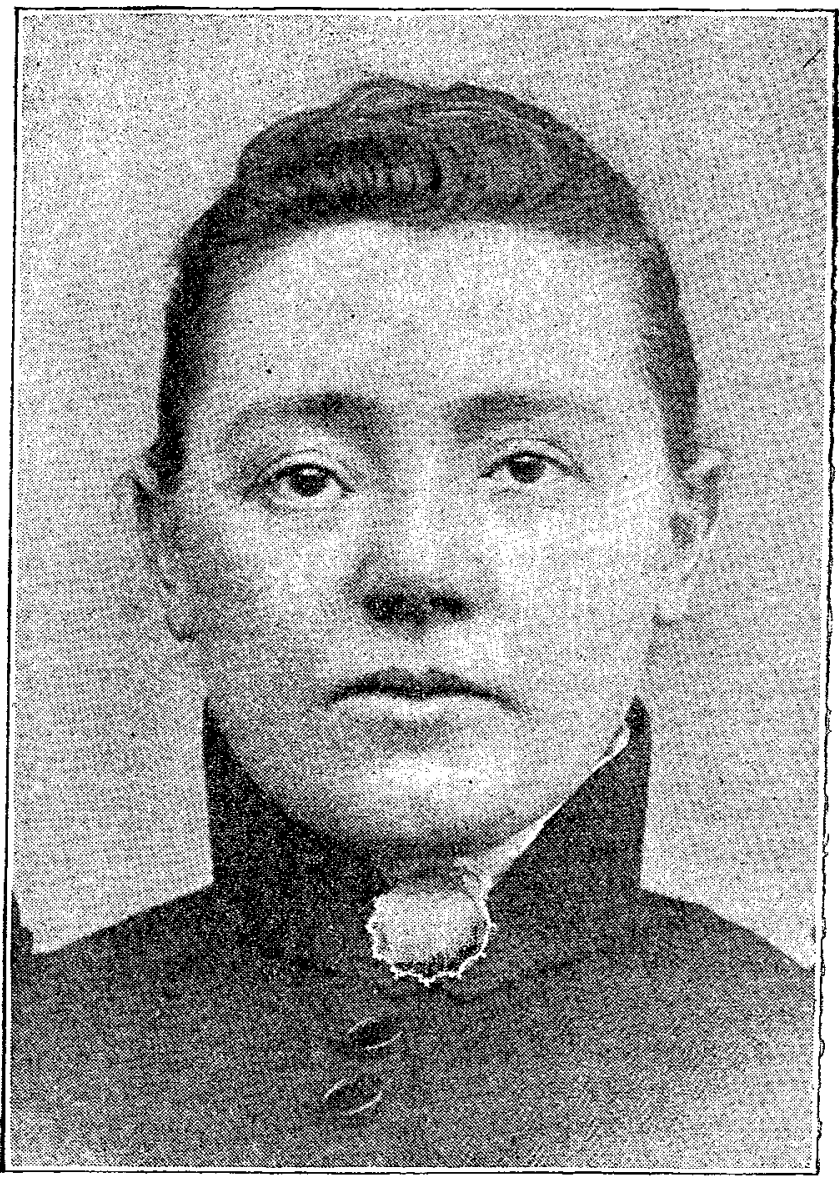

of the orbit. The patient did remarkably well, and now, two years after the operation, the nose remains free. The eye has gone quite back to the middle line, though it has not quite recovered its proper level (Fig. 2). There is slight hyperresthesia round the orbit. The patient was seen by the late Sir W. Bowman with Mr. Morton before she came under my care, and he pronounced it a case of suppuration in the posterior ethmoidal cells. The case was shown at the February meeting of the North London Medico-Chirurgica? Society.

Devonsbire-street, $\mathbf{w}$.

The Bristol Eye Hospital. - A new wing to this institution was formally opened by the Duchess of Beaufort on April 20th. The building comprises three storeys, and every care has been taken to promote the comfort of the blind and partially blind. To the wing an annexe has been added fitted with lavatories and other conveniences specially adapted for the blind. Two summer houses have been erected on the adjoining lawn. The interior of the building is painted in light colours, white and yellow predominating, thus making the rooms as cheerful as possible for the inmates. 